\title{
LOS PROCESOS COGNITIVOS EN LA COMPRENSIÓN Y ABSTRACCIÓN DE LOS APRENDIZAJES
}

\author{
Mercedes Fabiola Moreno Benítez* \\ Maricela Sánchez Espinoza*
}

\section{RESUMEN}

Una de las necesidades básicas de la educación en el futuro será preparar a los alumnos para la Sociedad del Aprendizaje, dado que la información y los "saberes" son el recurso más crítico para el desarrollo social y económico, de ahí que las recomendaciones a nivel mundial sean desarrollar habilidades cognitivas para poder acceder a la información.

La educación en este sentido exige trasformar las prácticas para garantizar la excelencia y así satisfacer las necesidades básicas de aprendizaje. La visión del cambio sobre el aprender y enseñar, supone un marco conceptual que armonice los conocimientos, las habilidades y los valores. Esta transformación el PEA (proceso enseñanza aprendizaje) cambia por PAE (proceso aprendizaje enseñanza), y bajo este marco nace el proyecto académico actual que propone identificar la comprensión en el desempeño que refleja el dominio de competencias.

Cada docente, cada formador de maestros ha de enfrentarse hoy a la pedagogía de las competencia; algunos se adhieren a ella totalmente, otros muestran cierta inquietud. Esto nos hace recordar que en pedagogía como en otras ciencias humanas, no existen verdades absolutas, es decir los conceptos empleados se cristalizan conforme evoluciona la comprensión sobre el mismo.

\section{ABSTRACT}

One of the basic needs of Education in the future will be to prepare students for the Society of Learning, since the information and "knowledge" are the most critical re- source for social and economic development, for that reason the recommendations at world-wide level are to develop mental abilities to be able to accede to information. 
C. Doctora, Docente en Posgrado de la ULSA Benavente $Y$ Coordinadora Académica de la Articulación en Educación Básica de la S.E.P.

Doctora en Educación, Docente investigadora de la ULSA Benavente. maricelasaem3@hotmail.com

Education, in this sense demands to transform the practices to guarantee the excellence and thus satisfy the basic needs of learning. The vision of change on learning and teaching, supposes a conceptual frame which harmonizes knowledge, abilities and values. This transformation of the PEA (teaching-learning process) changes by PAE (learning-teaching process), and under this frame the present academic project is born, it proposes to identify the understanding in the performance that reflects the dominion of competences.

Each teacher, each educator's trainers have to face the Pedagogy of the competence today; some adhere to it totally, others show certain restlessness. This makes us remember that in pedagogy like in other Human Sciences, absolute truths do not exist, that is to say, that the used concepts are crystallized as the understanding on the same evolves.

\section{MARCO TEÓRICO}

\section{Qué es la sociedad de la información}

La sociedad industrial se sustentaba en el uso de los recursos clásicos de la economía; la sociedad de la información se fundamenta en el capi- tal humano reforzado por las nuevas tecnologías. Hoy el conocimiento se renueva cada cinco años y en ese lapso se genera más información que en todos los cientos de años previos. Esta transformación conduce a que la educación se plantee de manera diferente.

Las principales características de la sociedad de la 
información son:

- La economía dirigida por conocimientos globales.

- La comunicación como directiva.

- El aprendizaje como fuente de un atributo sostenido y competitivo.

- La información compartida contra el atesoramiento del conocimiento.

La sociedad de la información exige que se diseñe un modelo de planeación estratégica del aprendizaje, junto con un modelo de administración del aprendizaje que nos permita la construcción de competencias. Las competencias entonces son la base de la nueva educación.

Es importante recordar que las competencias parten del marco conceptual que provee la institución con su misión y filosofía características.

Para elegir el núcleo de competencias básicas que se requiere construir, es necesario analizar y responder en forma realista las siguientes preguntas:

1. ¿Cuáles son las competencias básicas y necesarias para obtener buenos resultados en la educación contemporánea?

2. ¿Qué indicadores permiten elegir las competencias que se van a construir?

3. ¿Cuáles son los medios más efectivos para construir estas competencias?

4. ¿Cuáles son los medios más efectivos para comprobar que los alumnos han construido estas competencias? 


\section{Las competencias ¿por qué? y ¿para qué?}

El maestro y el alumno debieran tener una mente bien orientada más que llena de datos. De partir de lo concreto y de la experiencia, de apoyarse de las cosas reales, del deseo de poner las manos en la masa, de construir el saber permitiendo así la adquisición de competencias.

Montaigne, 1580.

Las modificaciones en el mundo del trabajo, sobre todo a causa de la introducción de las nuevas tecnologías, generan nuevas necesidades formativas, ante las cuales el aula y las instituciones de formación se muestran impotentes para su satisfacción. Más allá de las reformas habidas y de su insuficiencia por la continua demanda socio laboral, ante el acelerado y progresivo cambio, se observa cómo las propias empresas pasan a constituirse en instituciones formativas, productoras de competencias y cualificaciones concretas e inmediatas.

La formación no puede consistir solamente en aprobar asignaturas tal y como se plantean en las instituciones educativas. Es necesario integrar conocimientos empíricos y prácticas cognitivas que lleven a los estudiantes a aprendizajes relevantes.

La competencia es construida por el individuo a partir de su experiencia en la vida profesional o personal (familiar, asociativa, política, etc.). Entonces ser competente es ser capaz de hacer elecciones, de negociar, de tomar iniciativas, de asumir responsabilidades, en pocas palabras ser capaz de ir más allá de lo prescrito y hasta de lo prescriptible. 
La necesidad del cambio está asumida en la educación superior desde hace tiempo, otra cosa es su implantación. Un ejemplo de ello es que hace algunos años, la Conferencia Mundial sobre la educación superior, convocada por la United Nations Educational, Scientific and Cultural Organization (UNESCO, 998) estableció que en el contexto económico, social y tecnológico, caracterizado por los cambios y la aparición de nuevos modelos de producción basados en el conocimiento y sus aplicaciones, así como por el tratamiento de la información, deben reforzarse y renovarse los vínculos entre enseñanza superior, el mundo del trabajo y otros sectores de la sociedad, para lo cual dicha conferencia trazó los siguientes lineamientos:

Combinar estudio y trabajo.

Intercambiar personal entre el mundo laboral y las instituciones de educación superior.

Revisar los planes de estudio para adaptarlos mejor a las prácticas profesionales.

Crear y evaluar conjuntamente modalidades de aprendizaje, programas de transición, de evaluación y reconocimiento de los saberes previamente adquiridos por los estudiantes.

Integrar la teoría y la formación en el trabajo.

En este sentido, el enfoque pedagógico por competencias es perfectamente compatible con la ciudadanía y la cultura. Al optar por esta vía, la escuela responde a la necesidad de dar sentido a los aprendizajes, a la constancia de que el alumno no es un recipiente que el maestro tiene la misión de llenar, sino una persona que construye esos conocimientos en función de lo que él es (sus conocimientos, sus intereses, etc.). Al adoptar una pedagogía para la construcción del saber y la adquisición de competencias, la escuela tiene la esperanza de reducir los "cono- cimientos muertos" a favor de "conocimientos vivos" mismos que bien integrados le servirán a lo largo de la vida.

Este cambio ha generado quejas de los maestros, por los desempeños insuficientes de los alumnos que se dan a conocer en las evaluaciones como ENLACE, PISA, CENEVAL, EXCALE. Pero, ¿qué se debe trabajar: aplicar, abstraer, restituir? 
El principio didáctico del enfoque por competencias propone otro camino. Adquirir una competencia es sencillamente "aprender a hacer lo que no se sabe, haciéndolo" esto es parafraseando a Platón, San Agustín y Meirieu. Este proceso exige la individualización de los aprendizajes, ya que debe propiciar una mayor conciencia sobre la heterogeneidad que hay en nuestras aulas, en otras palabras, aprender en la diversidad y encontrar riqueza en este intercambio. Por su naturaleza, el enfoque por competencias abre el camino para que el profesor brinde experiencias metodológicas al alumno, y en la práctica deberá facilitar la evaluación formativa, así ofrecer una visión positiva del error.

Pero ¿a qué finalidades obedece este cambio? ¿Qué tipo de hombre y mujer se desea formar? ¿un ser que recite textos? O bien ¿una persona dotada de herramientas de reflexión que le permita abordar los mismos textos, emprendiendo una investigación personal de su sentido? Mujeres y hombres capaces de tomar distancia frente a los discursos dominantes? ¿Individuos aptos para resolver situaciones complejas?

El concepto de competencia se cristaliza de varias formas, por ejemplo:

a) como el estrecho sentido de "saber ejecutar", es decir: hacer una resta, escribir un participio pasado, elaborar una hipótesis sobre el sentido de una palabra desconocida, etc.

b) otros las consideran como la capacidad de afrontar una situación nueva y compleja movilizando varios "saberes y haceres", como: elaborar una estrategia de comunicación, un saber histórico, investigar y enunciar correctamente propiedades geométricas a partir de situaciones concretas, aprender a diseñar modelos, etc.

...Una competencia implica un saber hacer (habilidades) con saber (conocimiento), así como la valoración de las consecuencias del impacto de ese hacer (valores y actitudes). La competencia es la puesta en juego de conocimientos, habilidades, actitudes y valores para el logro del propósito en un contexto dado. (Plan de estudios, 2006. Educación básica. Secundaria. SEP, p. ） 


\section{El significado de aprender}

La educación basada en competencias es un enfoque sistemático del conocer y del desarrollo de habilidades; se determina a partir de funciones y tareas precisas. Se describe como un resultado de lo que el alumno está capacitado a desempeñar o producir al finalizar una etapa. La evaluación determina, qué específicamente va a desempeñar o construir el estudiante y se basa en la comprobación de que el alumno es capaz de construirlo o desempeñarlo.

Por ejemplo: El piloto de un avión de línea se encuentra, durante un vuelo, ante una gama de situaciones que pueden ir desde la sumisión estricta a consignas, hasta situaciones únicas e inéditas, caracterizadas por la incertidumbre, y cuya solución depende de su actividad de comprensión. En un avión de línea, las fases de despegue y de aterrizaje serán las más procedimentales.

La competencia permite comparar entre capacidad de afrontar una situación (ejecución abierta) y la ejecución de un plan reconocido (ejecución cerrada).

Cuando el cursor de la competencia se encuentra cerca de las situaciones de prescripción escrita, la competencia se define en términos de saber-hacer. En efecto, se reduce a ejecutar una operación o un conjunto de operaciones. A cumplir instrucciones, a respetar estrechamente las consignas. Esta definición corresponde al enfoque Tayloriano, y el saber hacer puede ser considerado como el grado elemental de la competencia.

Por el contrario, cuando el cursor de la competencia está en la prescripción abierta, la competencia se define en términos de saber actuar y reaccionar. En estas circunstancias ser competente es: saber qué hacer y cuándo. Ante los imprevistos y los azares, ante la complejidad de los sistemas, y las lógicas de la acción se deben tomar iniciativa y decisiones, negociar, arbitrar, hacer elecciones, correr riesgos, reaccionar ante descomposturas, innovar lo cotidiano y asumir responsabilidades. 
Para ser competente no basta con ejecutar lo prescrito, hay que ir más allá de lo prescrito. Para nosotros, en el mundo escolar, las preguntas sobre las competencias disciplinarias terminales son: ¿se ubican en lo prescrito o más allá de lo prescrito?

La pedagogía de las competencias parte de comparar el desempeño de los novatos y los expertos; éstos últimos perciben más pronto y pro- fundamente. Pasan relativamente más tiempo analizando el problema antes de actuar, y formulan representaciones más complejas de la tarea. En el ámbito de su experiencia poseen un repertorio de conocimientos procesuales más poderosos y mejor automatizados. Ejercen un control metacognitivo (monitoring) sobre su avance y sobre la tarea.

La competencia en la educación, es una convergencia de los comportamientos sociales, afectivos y las habilidades cognoscitivas, psicológicas, sensoriales y motoras que permiten llevar a cabo adecuadamente un papel, un desempeño, una actividad o una tarea.

Gardner (998), por ejemplo, en su Teoría de las Inteligencias Múltiples distingue de la siguiente manera las competencias que deben desarrollar los alumnos en el área del arte:

-Producción: Hacer una composición o interpretación musical, realizar una pintura o dibujo, escribir imaginativa o creativamente.

-Percepción: Efectuar distinciones o discriminaciones desde el pensamiento artístico.

-Reflexión: Alejarse de la propia producción e intentar comprender los objetivos, motivos, dificultades y efectos conseguidos.

La construcción de competencias no puede realizarse de manera aislada, sino que debe hacerse a partir de una educación flexible y permanente, desde una teoría explícita de la cognición, dentro del marco conceptual de la institución, en un entorno cultural, social, político y económico.

La educación por competencias se refiere a una experiencia práctica, que necesariamente se enlaza a los conocimientos para lograr un fin. La teoría y la experiencia práctica se vinculan, utilizando la primera para aplicar el conocimiento a la construcción o desempeño de algo. 
Desde el currículum, la educación basada en competencias se concentra en:

- Los conocimientos.

- Las habilidades.

- Las actitudes inherentes a una competencia (actitudes o comportamientos que respondan a la disciplina y a los valores).

- La evaluación de los logros mediante una demostración del desempeño o de la elaboración de un producto.

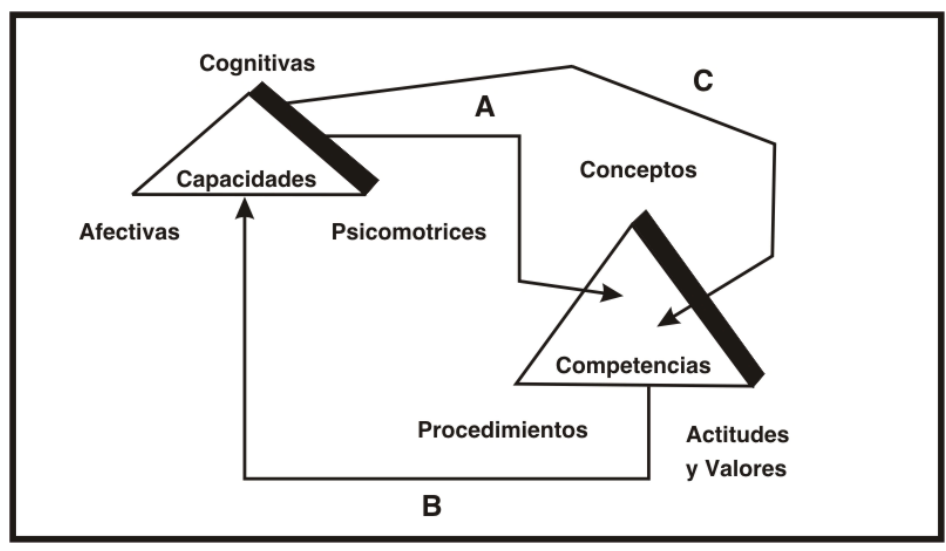

Figura Caracterización de las competencias (Fernández, 997, p.3)

Las competencias sólo son definibles en la acción (Tejada, 1999 a 2002,

2004). En la línea de lo apuntado anteriormente, las competencias no son reducibles ni al saber, ni al saber hacer, por tanto, no son asimilables a lo adquirido en formación. Poseer unas capacidades no significa ser competente. Es decir, la competencia no reside en los recursos (capacidades), sino en la movilización misma de los recursos. Para ser competente es necesario poner en juego el repertorio de recursos. Saber, además, no es poseer sino utilizar. 


\section{METODOLOGÍA}

El objetivo principal de un trabajo de investigación realizado con estudiantes de Licenciatura en Educación, consistió en valorar las tareas intelectuales básicas utilizadas para entender y comprender la información que se les presenta; así como los procesos cognitivos que se desarrollan al abordar los contenidos de aprendizaje. Esta investigación hace énfasis en los procesos intelectuales, como el análisis, la crítica y la reflexión; procesos que el propio estudio busca explicar para pro- mover en los estudiantes una enseñanza basada en un pensamiento disciplinar, que les permita estudiar a fondo temas sustanciales de las asignaturas elementales, utilizando formas de pensamiento que los acerque a comprender el mundo que los rodea.

Con estos elementos de estudio de la investigación, es posible preguntarse: ¿Qué tipos de procesos de pensamiento emplean los estudiantes para comprender lo que aprenden? ¿Cuáles son las tareas intelectuales necesarias para que los estudiantes comprendan lo que están aprendiendo? El análisis, la crítica y la reflexión ¿son procesos de pensamiento que facilitan la comprensión de los aprendizajes? Desde el tratamiento y abordaje de los contenidos de aprendizaje ¿pueden los estudiantes desarrollar habilidades de comprensión y abstracción? Entre los criterios para observar y comprender los procesos de pensamiento en el aprendizaje, se encuentran la observación y el desarrollo de ciertas tareas cognitivas elementales en la resolución de problemas a través de cuestionamientos simples y complejos, que permitirán elaborar los conceptos necesarios para extraer y comprender mejor lo trabajado en clase.

La idea de ir conociendo y señalando estos procesos es con la intención de acercarse a lograr niveles de comprensión y abstracción en los estudiantes; de tal forma que el aprendizaje adquiera significado. 
Se decidió estudiar los procesos de pensamiento que los estudiantes de la Licenciatura en Educación emplean, en la comprensión y abstracción de contenidos de aprendizaje, bajo la selección de un estudio de caso único, que permite indagar desde la observación y las entrevistas semiestructuradas los distintos procesos empleados por los estudiantes cuando se acercan a comprender y abstraer la información, La diversidad de conceptos y significados en la clase. Por ello se selecciona el estudio intrínseco de caso.

El estudio cualitativo de caso, tuvo como parte fundamental la interpretación fundamentada, que permitió precisar o sustanciar ciertos significados de las observaciones y de otros datos recogidos desde los actores (personas estudiadas); así como los lugares y circunstancias que "mejor" ayudaron a comprender la realidad estudiada.

En la medida en que se fueron construyendo ciertos significados, sacando conclusiones y revisando lo que los autores señalan, se crearon categorías de análisis propias al estudio. Entre ellas conviene señalar las siguientes:

Procesos cognitivos: se refieren a todas aquellas organizaciones internas que existen en la mente de las personas como el conocer, interpretar y ampliar el conocimiento partiendo de juicios afinados de la realidad percibida.

2. Comprensión en el aprendizaje: la comprensión implica un conjunto de procesos que los estudiantes llevan a cabo como el análisis, la crítica y la reflexión; así como el elaborar juicios afirmativos para emprender síntesis y crear productos que incorporen principios o conceptos centrales para una disciplina.

3. Abstracción: la abstracción surge como resultado de aquellos conceptos que al ser fijados conscientemente y grabados en la memoria pueden ser recuperados con facilidad ayudando a conectar una nueva experiencia de aprendizaje que podrá aplicarse en otras situaciones similares, puesto que el concepto ha de ser un instrumento del pensamiento y no simplemente un fragmento de saber. 
4. Aprendizaje: es un proceso activo que corresponde sólo a los alum nos y únicamente mediante esta actividad es como las actitudes y los hábitos se forman, las habilidades y destrezas se desarrollan y existe apropiación de información científica construyéndose el carácter.

Para formular estas categorías, el estudio utilizó como procedimiento el análisis cualitativo que permitió cuestionar a los profesores respecto a los procesos de pensamiento que emplean con los estudiantes para favorecer las habilidades de comprensión y abstracción. Además se llegó a la interpretación y comprensión de situaciones que los estudiantes experimentan en el desarrollo de las clases y que en términos de significado suceden en este contexto escolar, a través de los comentarios de los propios sujetos.

Los actores del caso fueron dieciocho estudiantes y cuatro profesores de la Licenciatura en Educación, a quienes se les presentó una descripción del trabajo previsto; logrando con ello el acceso y el permiso requerido para el desarrollo de la investigación.

En esta investigación de tipo cualitativo se llegó a una reflexión profunda de finos detalles, de las actitudes y del significado que se le daban a las interacciones que se observaron de los acontecimientos claves; haciendo un registro cuidadoso de lo que acontecía mediante notas de campo y un listado de procesos cognitivos, surgidos de las tareas intelectuales que realizan los estudiantes en el abordaje de contenidos, informaciones presentadas y experiencias de aprendizaje. La reflexión analítica a partir de los registros realizados y de los procesos cognitivos observados, permitieron realizar una descripción detallada de lo observado.

\section{Resultados y desempeño}

Para expresar los propósitos educativos, según recomienda The Concepts and Methods of the Competency Outcomes and Performance Assessment, es preferible utilizar, en vez del vocablo tradicional "objetivos", el término más actual "resultados", porque define con mayor claridad las metas del aprendizaje y las prácticas básicas y avanzadas de la disciplina. El término objetivo implica intención y el término competencia implica resultado. 
"Resultados" son los frutos que se obtienen, el final del producto, el foco de todas las actividades relacionadas; requieren que se hayan construido competencias y desarrollado las habilidades que les con- ciernen, además de que el estudiante se haya comprometido y hecho competente en las habilidades que utilizará en su práctica profesional. Lo encontrado en el estudio se refiere a las tareas cognitivas que se pueden seguir al abordar los contenidos de aprendizaje para que la comprensión y abstracción pueda darse desde los estilos cognitivos de los estudiantes (listado de tareas cognitivas encontradas para la comprensión y abstracción de los aprendizajes).

- Hacer preguntas relevantes del contenido que se trabaja

- Precisar y concretar ideas relacionadas con la información que se procesa y abstrae

- Construir significados a partir de las ideas de otros

- Aceptar la crítica razonable

- Escuchar los puntos de vista desde el nivel de comprensión

- Ofrecer analogías apropiadas en relación al contenido que se trabaja

- Clarificar conceptos

- Establecer distinciones y condiciones relevantes al integrar significa dos o conceptos

- Fundamentar opiniones con razones convincentes

- Proporcionar ejemplos y contraejemplos

- Sacar inferencias apropiadas de lo que se está elaborando o construyendo

- Realizar juicios evaluativos respecto a la información que se abstrae.

Estas competencias se valoraron a través de procesos cognitivos que realizan los estudiantes para construir, reconstruir y elaborar el cono- cimiento.

Este enfoque por competencias está contribuyendo a transformar los procesos de enseñanza-aprendizaje, contextualiza la formación, orienta la organización de los contenidos, promueve la formación integral (integra el saber conocer con el saber hacer, el saber ser y convivir) y establece mecanismos de evaluación permanentes y de rigurosidad basados en el desempeño ante situaciones problemáticas del contexto (disciplinar, social, científico, etc.). 
Los aspectos innovadores de la formación basada en competencias, son, entre otros, los siguientes:

1. El reconocimiento de los aprendizajes, independientemente del con texto en el cual se hayan adquirido.

2. La integración entre teoría y práctica.

3. El énfasis en el desempeño real ante situaciones y problemas de la vida cotidiana, la investigación y el entorno profesional.

4. La articulación del saber ser con el saber conocer, el saber hacer y el saber convivir.

5. El establecimiento de procesos de gestión de calidad para asegurar el logro de los aprendizajes esperados en los estudiantes a partir de la autoformación y la capacitación de los docentes y de los administradores en el ámbito de la educación.

La diversidad considera los diferentes estilos de aprendizaje presentes en el ser humano, que han sido objeto de estudio en el campo de la educación y han servido para iniciar cambios significativos en el proceso enseñanza-aprendizaje. Varias universidades hacen investigación sobre preferencias perceptuales. Pero en general el objetivo es identificar los diferentes estilos de aprendizaje y adecuar las prácticas de enseñanza para favorecer el desempeño de los estudiantes, diseñando estrategias con el fin de lograr el perfil de aprendizaje (Sternberg, 999; Zhang, 2002). Actualmente el aprendizaje está pensado para el desarrollo de competencias; la propuesta es reconocer el estilo de aprendizaje de cada individuo y adecuar la enseñanza en el aula, es decir tener una conexión lógica para lograr un aprendizaje efectivo (Burke y Dunn, 2002). 
En estudios recientes se ha encontrado que los estilos existentes en los estudiantes van desde el reflexivo o autocrítico, al creativo o práctico y al receptivo 0 constructivo. Estos estilos de pensamiento promueven el aprendizaje cooperativo, el cual es esencial para que los estudiantes aprendan más trabajando en grupo.

A la escuela le queda el reto de tomar como método la innovación, (Giroux, 990), entonces asumamos el compromiso de cambiar, primero nosotros y después a la institución adecuándola para esa tarea. Esa innovación supone generar un currículo de base centrado en:

- Capacidad de búsqueda, procesamiento y abstracción de la información.

- Capacidad de adaptación o cambio sistemático.

- Capacidad creativa, a partir de una enseñanza con diferentes lógicas.

\section{CONCLUSIONES}

Lo nuevo, en el ámbito de la sociedad cognitiva, resulta ser la necesidad de universalizar el conocimiento y el dominio de los códigos de interpretación; democratizar el conocimiento cuestionador favorece la iniciativa individual y colectiva. Éste es el reto de la sociedad y la escuela en el trabajo por competencias.

Educar hoy, representa un compromiso con la vida real y ayudar, con otros, a construir el futuro para otros. Educar exige antes que un inventario de respuestas, hacer bien las preguntas.

Una de las mejores maneras de enseñar de forma dialogada es animar a los chicos a que hagan preguntas (Sternberg, 999:38). Los estudiantes son preguntones por naturaleza, tienen que aprender el modo de adaptarse a un modo complejo y en continua transformación. Pero que sigan haciendo preguntas $y$, sobre todo, que sean buenas preguntas dependerá en buena parte de la forma en que los adultos respondan a las preguntas. La capacidad de formular buenas preguntas y de saber contestarlas es una parte fundamental de la inteligencia, podría incluso considerarse como la más importante, se trata pues de una actitud que podemos fomentar $o$ reprimir.

\section{NOTAS}

Denyer y otros (2007). Las competencias en la educación. Dice: Lo que las empresas necesitan son personas con iniciativa y capaces de tomar decisiones a nivel local, para enfrentar situaciones imprevistas. El término competencias reemplaza al de capacitación 


\section{FUENTES DE CONSULTA}

- BRUNER, G. ( 998), Actos de significado, más allá de la revolución cognitiva. Psicología y educación. México: Alianza.

- COLL, G. Santos Torres ( 998), El marco curricular en una escuela re- novada. Madrid: Popular.

- DEWEY, J. ( 998), Cognición y desarrollo humano. Barcelona: Paidós

- EISNER, Elliot W. ( 998), El ojo ilustrado. Indagación cualitativa y mejo- ra de la práctica educativa. Barcelona: Paidós Educador

- ESTÉVEZ, E. (2002), Enseñar a aprender. Estrategias cognitivas. Barce- lona: Paidós

- GARDNER, Howard (2000), La educación de la mente y el conocimiento de las disciplinas.España:Paidós

- GARDNER, Howard ( 997), Inteligencias múltiples: la teoría en la prác- tica. Barcelona:Paidós.

- KLINGLER, C. y Vadillo, G. (2000), Psicología cognitiva: estrategias en la práctica docente. México: McGraw-Hill

- LOPEZ Calva, M. ( 998), Pensamiento crítico y creativo en el aula. México:Trillas.

- MARTíNEZ Beltrán, J. M. (2007), La mediación en el proceso de aprendizaje. España: Bruño

- MEECE, J. (2000), El desarrollo cognoscitivo: las teorías de Piaget y Vigotsky y desarrollo cognitivo. Compendio para educadores. México: Oc- taedro

- NICKERSON, R. S., Perkins D., Smith E. ( 998), Enseñar a pensar aspec- tos de la aptitud intelectual. Barcelona: Paídos

- STERNBERG, R. (2003). Inteligencia exitosa. Barcelona: Paidós

- STERNBERG, R. ( 999), Estilos de pensamiento. Barcelona:Paidós

- TAYLOR, S. J., R. Bogdan ( 998). Introducción a los métodos cualitativos de investigación. Barcelona:Paidós 